\title{
On Concurrency Control in Sliding Window Queries over Data Streams*
}

\author{
Lukasz Golab ${ }^{\dagger}$ Kumar Gaurav Bijay ${ }^{\ddagger}$ M. Tamer Özsu ${ }^{\dagger}$
}

Technical Report CS-2005-28

December 2005

\section{Waterloo}

${ }^{*}$ This research is partially supported by the Natural Sciences and Engineering Research Council (NSERC) of Canada and Communications and Information Technology Ontario (CITO).

${ }^{\dagger}$ School of Computer Science, University of Waterloo, Waterloo, Ontario N2L 3G1, Canada, $\{$ lgolab,tozsu\}@uwaterloo.ca

${ }^{\ddagger}$ Department of Computer Science and Engineering, Indian Institute of Technology, Bombay, India, gauravk@cse.iitb.ac.in. Work done while the author was visiting the University of Waterloo. 


\begin{abstract}
Data stream systems execute a dynamic workload of long-running and one-time queries, with the streaming inputs typically bounded by sliding windows. For efficiency, windows may be advanced periodically by replacing the oldest part of the window with a batch of newly arrived data. Existing work on stream processing assumes that a window cannot be advanced while it is being accessed by a query. In this paper, we argue that concurrent processing of queries (reads) and window-slides (writes) is required by data stream systems in order to allow prioritized query scheduling and improve the freshness of answers. We prove that the traditional notion of conflict serializability is insufficient in this context and define stronger isolation levels that restrict the allowed serialization orders. We also design and experimentally evaluate a transaction scheduler that efficiently enforces the new isolation levels by taking advantage of the access patterns of sliding window queries.
\end{abstract}

\title{
1 Introduction
}

A Data Stream Management System (DSMS) executes two types of queries-long-running and snapshot - whose input streams are typically bounded by sliding windows. Long-running queries return updated answers periodically and often involve complex aggregation for monitoring purposes. For instance, an Internet service provider (ISP) may issue queries over a window of recently collected IP packet headers in order to track bandwidth usage, monitor network performance, or detect malicious attacks. These queries may include complex aggregates such as top- $k$ lists of "heavy" users, destinations, protocol types or port numbers, quantiles over packet round-trip times or packet lengths, and COUNT DISTINCT queries over IP addresses requesting a connection $[9,11]$. Snapshot queries are analogous to traditional database queries in that they can be submitted to the DSMS at any time, are executed once (when posed), and return an answer over the current state of the inputs. Snapshot queries may be used to obtain further details in response to a change in the result of a long-running query. For example, the ISP may ask more detailed queries about a particular host or link that appears to be malfunctioning.

Previous work on sliding window query processing $[3,8,14,17,19]$ and stream query languages $[1,2,6]$ assumes that windows slide periodically by replacing the oldest part of the window with a batch of fresh data. A periodically-sliding window can be modeled as a circular array of sub-windows, with each sub-window spanning an equal time interval for time-based windows (e.g., a ten-minute window that slides every minute) or an equal number of tuples for tuple-based windows (e.g., a 100-tuple window that slides every ten tuples). We define a window update as the process of replacing the oldest sub-window with newly arrived data, thereby sliding the window forward by one sub-window. We will use the terms window update, window movement, and window-slide interchangeably.

As the windows slide forward by way of periodic updates, a DSMS executes a dynamic workload of long-running and snapshot queries. For now, suppose that query execution involves scanning a window, one sub-window at a time (we will discuss this in more detail in Section 2). Combined with periodic window movements, we can model DSMS data access in terms of two atomic operations: sub-window scan (read) and replacement of the oldest sub-window with new data (write). Thus, a window update is a single write operation, whereas a query is a sequence of sub-window read operations such that each sub-window is read exactly once. 
(a)

(b)

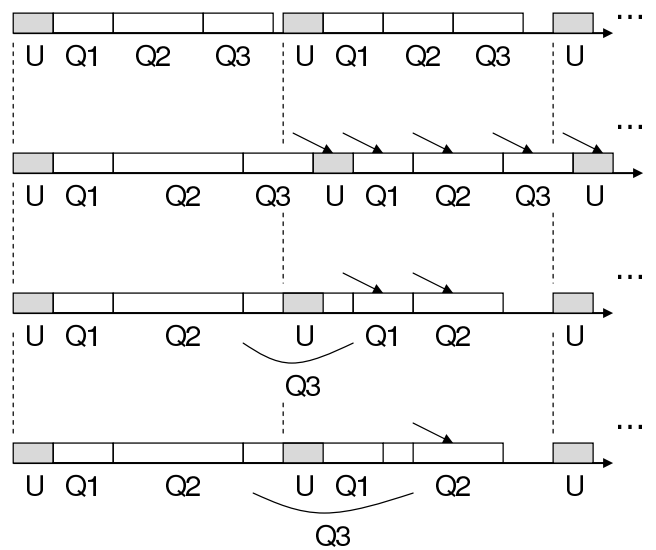

Figure 1: Examples of query and window update sequences in a DSMS.

A window may be scheduled to slide while being accessed by a query, resulting in a read-write conflict. Consider a sequence of operations illustrated in Figure 1 (a), where the processing times of window updates $(U)$ and queries $\left(Q_{1}, Q_{2}\right.$, and $\left.Q_{3}\right)$ are shown on a time axis. This represents an ideal scenario, where it is possible to execute all three queries between every pair of window updates, thereby avoiding read-write conflicts. However, the system environment, such as the query workload, stream arrival rates, and availability of system resources, can change greatly during the lifetime of a long-running query. Thus, a more realistic sequence is shown in Figure 1 (b), where $Q_{2}$ takes longer to execute than expected. $Q_{3}$ is still running when the second update is ready to be applied, causing a delay in performing the update, and, in turn, causing another read-write conflict when $Q_{3}$ is re-executed and the third update is about to take place. A similar problem arises if the system must process additional snapshot queries or if new long-running queries are deployed.

It may appear that read-write conflicts can be prevented by increasing the time interval between window updates, i.e., the sub-window size. However, all sub-windows must have the same size so that the overall window size is fixed at all times. Therefore, either the system must be taken off-line to re-partition the entire window, or two sets of sub-windows must be maintained during the transition period until the window "rolls over" and all the sub-windows have the new size. The first case is inappropriate for an on-line DSMS, whereas the second solution does not immediately eliminate read-write conflicts as concurrent queries and updates may still occur during the transition period.

Existing data stream solutions avoid read-write conflicts by serially executing queries and window movements. In other words, a query locks the window that it is scanning in order to prevent concurrent window movements. Interleaved execution of updates while a window is being scanned by a query is advantageous, provided that the following issue is resolved. Consider suspending the processing of $Q_{3}$ in order to perform a window update, as in Figure 1 (c). Recall that each query is assumed to perform a sequence of atomic sub-window reads, therefore it may be interrupted after it has read one or more sub-windows. It must be ensured that when resumed, $Q_{3}$ can correctly read the updated window state. If so, then the answer of $Q_{3}$ is slightly delayed (by the time taken to perform the update), but it is more up-to-date because it reflects 
the second update as well as the first. Otherwise, we are worse off than in Figure 1 (b), because the answer of $Q_{3}$ is delayed, but it is still not up-to-date. Another example is illustrated in Figure $1(\mathrm{~d})$, where $Q_{3}$ is suspended not only to perform a window update, but also to run $Q_{1}$ immediately afterwards. This is desirable if $Q_{1}$ is an important query that requires an immediate and up-to-date answer.

This paper studies concurrency control issues in a DSMS with periodic window movements, periodic executions of long-running queries, and on-demand snapshot querying. Our goal is to provide query scheduling flexibility and guarantee up-to-date results. The particular contributions of this paper are as follows.

- By modeling window movements and queries as transactions consisting of atomic subwindow reads and writes, we extend traditional concurrency theory to cover queries over periodically-advancing windows. We show that conflict serializability is not sufficient in the presence of interleaved queries and window movements because some serialization orders produce incorrect answers.

- We propose two isolation levels that are stronger than conflict serializability in that they restrict the permissible serialization orders.

- We design a transaction scheduler that efficiently enforces the desired isolation levels. The main idea is to exploit the access patterns of queries and window updates. The scheduler is proven to be optimal in the sense that it aborts the smallest possible number of transactions while allowing immediate (optimistic) scheduling of window updates.

- We perform an experimental evaluation of the transaction scheduler under various query workloads and system parameters, showing improved query freshness and response times with a minimal drop in throughput.

The remainder of this paper is organized as follows. Section 2 motivates and explains our system model and assumptions. Section 3 defines new isolation levels for DSMS transactions, and Section 4 presents a transaction scheduler for enforcing them. Section 5 presents experimental results, Section 6 compares the contributions of this paper to previous work, and Section 7 concludes the paper with suggestions for future work.

\section{Motivation and Assumptions}

\subsection{Data and Query Model}

A data stream consists of relational tuples with a fixed schema. Without loss of generality, we assume that each stream is bounded by a time-based window. A window of time-length $n t$ is stored as a circular array of $n$ sub-windows, each spanning a time-length of $t$ (each window may have different values for $n$ and $t$ ). Every $t$ time units, the oldest sub-window is replaced with a buffer containing incoming tuples that have arrived in the last $t$ time units. Additionally, the DSMS may materialize intermediate results of selected queries or sub-queries, e.g., sliding

window joins [8], which may also be stored as arrays of sub-windows [16]. We assume that $t$ 
is significantly larger than the time taken to perform a window update (otherwise, the system would spend all of its time advancing the windows rather than executing queries).

Given that long-running queries are used for monitoring purposes, they typically compute aggregates over a single window or a join of several windows; a selection predicate may precede the aggregate and a group-by condition may follow it. Each long-running query $Q$ also specifies its desired re-execution frequency. The frequency must be a multiple of $t$, i.e., $Q$ will be scheduled for re-execution every $m$ window updates, where $1 \leq m<n$. The DSMS attempts to execute all the queries with the desired frequencies, but it cannot guarantee that this will be the case at all times due to unpredictable system conditions.

We classify DSMS query execution strategies into four general types: window scan (WS), incremental scan (IS), summary scan (SS), and incremental summary scan (ISS). To illustrate them, suppose that two sums over the same attribute (and stream) are being computed, sum1 over a window of size $6 t$ (six sub-windows) and sum2 over a window of size $10 t$ (ten sub-windows).

WS is a default access path that scans the entire window (or windows), one sub-window at a time, and computes a query from scratch. As shown in Figure 2 (a), if sub-windows are read from youngest to oldest, then the sum over the shorter window may be re-used when computing the sum over the longer window.

One way to speed up the execution of some types of queries is to store permanent state that allows answers to be refreshed incrementally. IS, shown in Figure 2 (b), stores the previously calculated answer of each query and a pair of pointers denoting the window over which the answer was computed (indicated by the dotted arrows). Upon re-evaluation, we scan (all the tuples in) only those sub-windows which have been added or expired since the last re-execution. To compute new sums, we add the sum of the tuples in the new sub-window (lightly shaded) and subtract the sum of expired tuples (darkly shaded). This strategy applies to subtractable queries such as SUM and COUNT [3], where the contribution of expired tuples may be subtracted from the stored answer (MIN and MAX are two examples of aggregates that are not subtractable). Furthermore, incremental computation of complex aggregates, such as median or COUNT DISTINCT, requires that each query store a list of distinct values occurring in its window and their multiplicities (it is not sufficient to store the previous answer). Observe that expired tuples are found in different sub-windows, depending on the window size of the query. Therefore, if the workload includes many sum queries over windows of different sizes, then the entire window may need to be scanned in order to re-compute all the answers, as in WS.

Rather than storing separate state per query, SS maintains shared window summaries. For associative aggregates, such as SUM, COUNT, MAX, or MIN, we pre-aggregate each sub-window $[3,19]$. An example is shown in Figure 2 (c), illustrating shared processing of two sums over different window sizes. To handle non-associative aggregates, such as median, top- $k$, and COUNT DISTINCT, each sub-window summary stores counts of all the distinct values occurring within $[13,14]$. Alternatively, approximate answers of complex aggregates may be computed by storing summaries that contain estimates of the distribution of values in each sub-window. Examples include Count-Min sketch [10] and Flajolet-Martin sketch [12]. Note that separate pre-aggregated values, sketches, or counters need to be store for each group if a query contains a group-by clause. In all cases, query evaluation involves merging sub-window summaries, from youngest to oldest.

Finally, ISS is a combination of IS and SS. As illustrated in Figure 2 (d), it stores window summaries as well as individual query state. To refresh the answer, we only need to look up the 
(a)
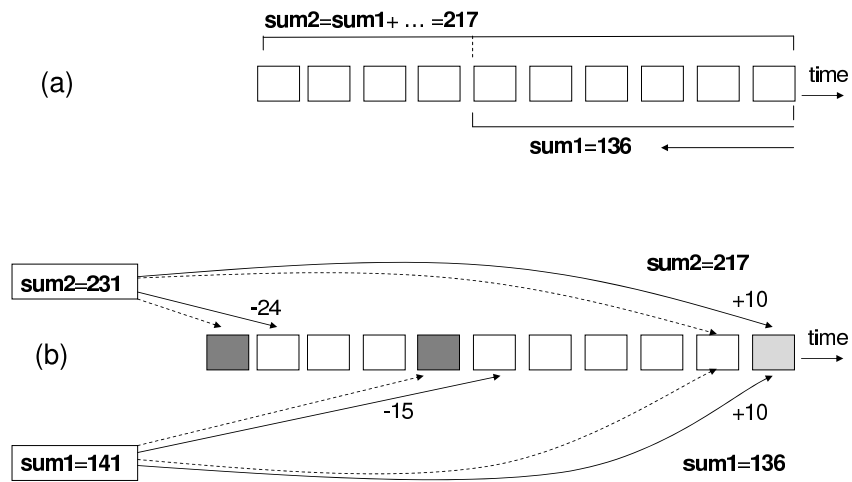

(c)
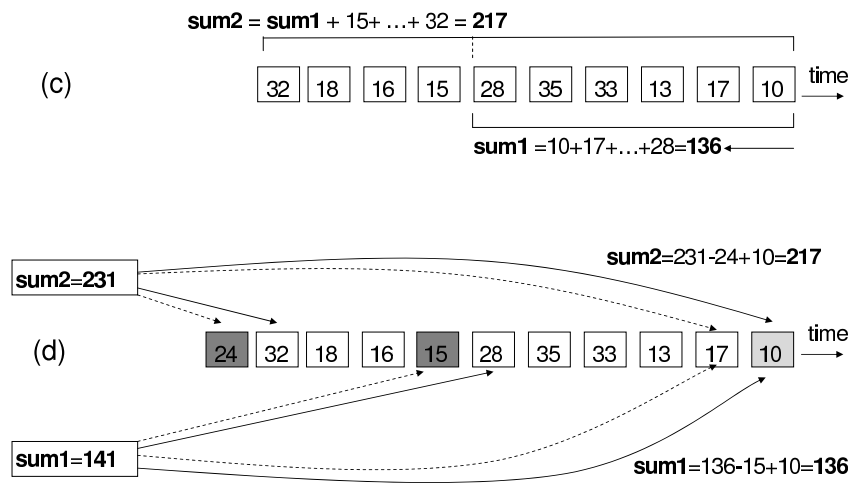

Figure 2: Four techniques for computing sliding window sums.

(pre-aggregated) sums of new and expired tuples. That is, only the summaries of new or expired sub-windows need to be accessed. As in IS, ISS is suitable only for subtractable queries.

\subsection{Motivation for Study of Concurrency Control}

In the remainder of this paper, we assume that WS and SS are used for query evaluation. First, WS is the default access plan for snapshot queries, which are not known ahead of time and therefore may not find any applicable summaries. Moreover, WS may be the only option for initial evaluation of a new long-running query; again, none of the stored answers or summaries may apply. In this case, the window may be scanned to produce the initial answer and optionally, to build a summary that the query can use for future re-executions (if the system has sufficient memory to store the summary).

Next, observe that SS and IS have comparable space usage, provided that the system workload includes similar queries over various window sizes. To see this, note that IS requires each query to store permanent state, even if many queries are identical except for their window sizes. For example, in Figure 2 (b), we cannot use sum2 to help compute sum1. On the other hand, SS 
stores one summary per sub-window, whose size is at most as large as the state of an individual query. In addition, IS must retain some expired sub-windows until each query has subtracted the contribution of expired tuples from its stored answer. In contrast, SS simply overwrites the oldest sub-window summary with a new summary. Moreover, SS yields faster processing times (IS must read all the sub-windows containing new or expired tuples) and may be used by non-subtractable queries.

Finally, note that the space usage of ISS (window summaries and query state) may be prohibitive. First, the number of summaries stored by the DSMS may be large. For example, if a query computes an average packet length with a group-by on protocol type, then we require separate sub-window sums and counts for each protocol type. Moreover, snapshot queries may need to suspend any long-running queries currently running, as in Figure 1 (d). In this case, we need to set aside state space for the suspended queries so that they can resume later, and reserve state space for any potential snapshot queries.

Recall Figure 1 (c) and (d). We need to allow a window to slide while being accessed by a query and ensure that the query reads the new window state correctly. In terms of concurrency control, we must not allow a query to have seen old tuples that have subsequently been expired. This problem does not exist in IS and ISS because a window update does not actually replace expired data. As discussed, expired sub-windows are retained until each query has subtracted the contribution of expired tuples from its stored answer. However, WS and SS both scan the window (or its summary), one sub-window at a time. Queries may see and old copy of the window if they have read an old sub-window that is about to be overwritten. We could retain expired sub-windows, but this requires more space and processing time (old sub-windows may need to be re-scanned by queries in order to remove the contribution of expired tuples from the answer currently being computed). Even then, it is desirable to prevent double-scanning whenever possible.

\subsection{System Model}

The assumed system architecture is illustrated in Figure 3. Let $w[i]$ denote the replacement of the $i$ th sub-window with newly arrived data, for $0 \leq i \leq n-1$. Each data stream generates periodic write-only transactions $T_{j}$ in subscript order, defined as $T_{j}=\left\{w_{j}[j \bmod n]\right\}$. They are processed by the transaction manager, which immediately propagates updates to all the summaries and materialized results that reference this window (e.g., new tuples are passed to the join operator, which probes the other window and generates new join results). For each stream, the transaction manager initially executes $T_{0}$ through $T_{n-1}$ to fill up the windows. Thereafter, each $T_{j}$ has the effect of moving the window forward by one sub-window. In order to ensure that queries have access to the latest data, the transaction scheduler executes each $T_{j}$ as soon as a buffer is full, thereby interrupting any concurrent queries.

Snapshot queries are executed by scanning a suitable summary, if available, or accessing the underlying window(s). Answers are returned in the form of a table. Long-running queries are re-executed periodically throughout their lifetimes and generate a stream of updated answers. A new long-running query is inserted into the query manager, or may be rejected if the system is overloaded. The query manager then determines an appropriate execution strategy for the new query, e.g., whether an existing summary may be used or a new summary should be built, 


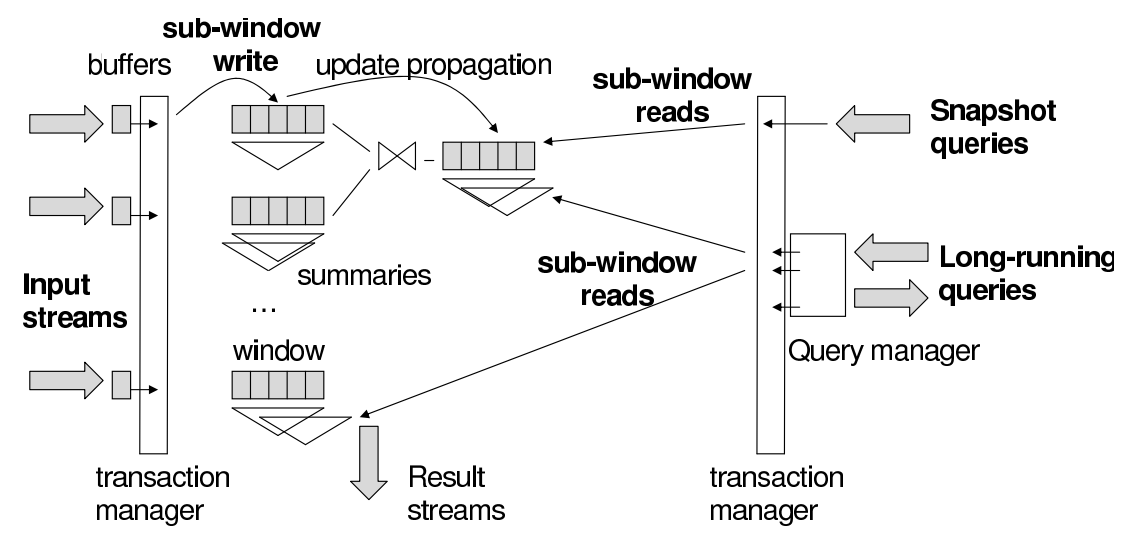

Figure 3: Assumed system architecture.

and whether the new query may be merged into a group of similar queries (possibly referencing windows of different lengths) for shared processing. The design of the query manager is an orthogonal topic, which we pursue in separate work. In this paper, we define an interface between the query manager and the transaction scheduler, which consists of read-only transactions corresponding to re-execution of one or several similar queries. We define $r[i]$ be a scan (read) of the $i$ th sub-window, or its summary, for $0 \leq i \leq n-1$ (without loss of generality, in the rest of the paper, we will refer to either of these as a sub-window). A snapshot query or a particular re-execution of one or more long-running queries is a read-only transaction $T_{Q k}$, defined as $T_{Q k}=\left\{r_{Q k}[0], r_{Q k}[1], \ldots, r_{Q k}[n-1]\right\}$. That is, each $T_{Q k}$ performs a scan of a window, sub-result, or summary, by reading each sub-window exactly once (queries over windows shorter than $n t$ may be defined similarly). We assume that sub-windows may be read in arbitrary order.

\section{Conflict Serializability in the Context of Sliding Window Queries}

\subsection{Serializability and Serialization Orders}

We begin by analyzing the isolation level requirements of queries over periodically-sliding windows. For clarity, we assume that queries access a single window and discuss queries accessing materialized sub-results in Section 4.3. First, we define the possible types of conflicts arising from concurrent execution of transactions. A conflict occurs when two interleaved transactions operate on the same sub-window and at least one of the operations is a write. Clearly, a readwrite conflict occurs whenever $T_{j}$ interrupts $T_{Q k}$, as in Figure 1 (c) and (d). This is because each $T_{Q k}$ reads every sub-window, including the sub-window overwritten by $T_{j}$. Technically, writewrite conflicts may occur if two write-only transactions that access the same sub-window are executed concurrently (i.e., $T_{j}$ and $T_{j+i n}$, for $i=0,1, \ldots$ ). However, we can ignore write-write conflicts due to our assumption of immediate execution of window movements. The traditional method for dealing with conflicts requires an execution history $H$ to be serializable. We show that serializability is insufficient in our context using the following example.

Assume a sliding window partitioned into five sub-windows, numbered zero through four, with sub-window zero being the oldest at the current time. Consider the following four histories- 


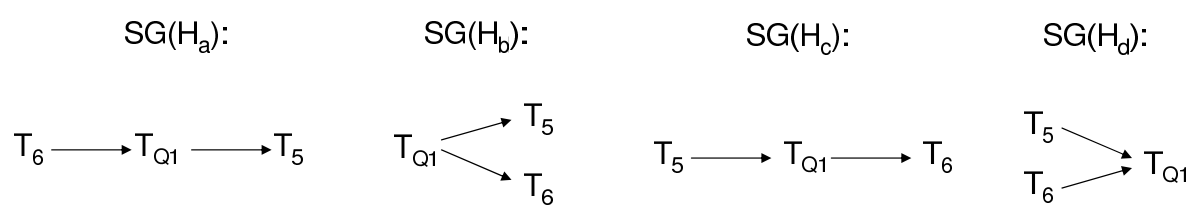

Figure 4: Serialization graphs for $H_{a}, H_{b}, H_{c}$, and $H_{d}$.

$H_{a}, H_{b}, H_{c}$, and $H_{d}$-with $c_{j}$ or $c_{Q k}$ denoting that transaction $T_{j}$ or $T_{Q k}$, respectively, has committed (for brevity, we omit the initial transactions $T_{0}$ through $T_{4}$ that fill up the window).

$H_{a}=r_{Q 1}[0] w_{5}[0] c_{5} w_{6}[1] c_{6} r_{Q 1}[1] r_{Q 1}[2] r_{Q 1}[3] r_{Q 1}[4] c_{Q 1}$

$H_{b}=r_{Q 1}[0] w_{5}[0] c_{5} r_{Q 1}[1] w_{6}[1] c_{6} r_{Q 1}[2] r_{Q 1}[3] r_{Q 1}[4] c_{Q 1}$

$H_{c}=r_{Q 1}[4] w_{5}[0] c_{5} r_{Q 1}[3] r_{Q 1}[2] r_{Q 1}[1] w_{6}[1] c_{6} r_{Q 1}[0] c_{Q 1}$

$H_{d}=r_{Q 1}[4] w_{5}[0] c_{5} r_{Q 1}[0] r_{Q 1}[3] r_{Q 1}[2] w_{6}[1] c_{6} r_{Q 1}[1] c_{Q 1}$

Each history represents interleaved execution of a read-only transaction $T_{Q 1}$ and two window movements, $T_{5}$ and $T_{6}$. Note that $H_{c}$ and $H_{d}$ reorder the read operations within $T_{Q 1}$; we will say more about ordering atomic operations in Section 4 . The associated serialization graphs are drawn in Figure 4. The direction of the edges corresponds to the order in which conflicting operations are serialized. In particular, there are two pairs of conflicting operations in each schedule: $r_{Q 1}[0]$ and $w_{5}[0]$, and $r_{Q 1}[1]$ and $w_{6}[1]$. Note that all four graphs are acyclic, therefore all four histories are serializable, but their serialization orders are different.

Let us analyze the data read by $T_{Q 1}$. For each history, consider the state of the sliding window shown in Figure 5, where the first sub-windows on the left $\left(s_{0}\right.$ through $\left.s_{4}\right)$ correspond to the initial state of the window after $T_{0}$ through $T_{4}$ were executed. Next, $T_{5}$ advances the window forward by one sub-window, which may be thought of as overwriting the old copy of sub-window $s_{0}$ (on the far left) with a new copy, appended after $s_{4}$. Thus, the state of the window after $T_{5}$ commits is represented by the contiguous sequence of sub-windows $\left\{s_{1}, s_{2}, s_{3}, s_{4}, s_{0}\right\}$. Then, $T_{6}$ advances the window again by appending a new copy of $s_{1}$ on the far right and implicitly deleting the old copy of $s_{1}$ on the left. Hence, the state of the window after $T_{6}$ commits is equivalent to the contiguous sequence of sub-windows $\left\{s_{2}, s_{3}, s_{4}, s_{0}, s_{1}\right\}$. Shaded sub-windows represent those which were read by $T_{Q 1}$ in each of the four histories, as explained next.

First, consider $S G\left(H_{a}\right)$ in Figure 4 and note that $H_{a}$ serializes $T_{6}$ before $T_{Q 1}$, meaning that the window movement caused by $T_{6}$ (creation of a new version of sub-window $s_{1}$ ) is reflected in the query. However, $H_{a}$ serializes an earlier window update $T_{5}$ after $T_{Q 1}$, therefore the prior window movement caused by $T_{5}$ (creation of a new version of $s_{0}$ ) is hidden from the query. Hence, $H_{a}$ causes $T_{Q 1}$ to read an old copy of $s_{0}$ and a new copy of $s_{1}$, as illustrated in Figure 5 (a), which does not correspond to a window state at any point in time. This is because the shaded rectangles do not form a contiguous sequence of five sub-windows. Next, recall that $H_{b}$ serializes both window movements after $T_{Q 1}$, therefore the query reads old versions of $s_{0}$ and $s_{1}$, as illustrated in Figure 5 (b). This corresponds to the state of the window after $T_{4}$ commits. By similar reasoning, $H_{c}$ allows $T_{Q 1}$ to read the state of the window after $T_{5}$ commits (recall Figure 5 (c)), and only $H_{d}$ ensures that $T_{Q 1}$ reads the most up-to-date state of the window that 


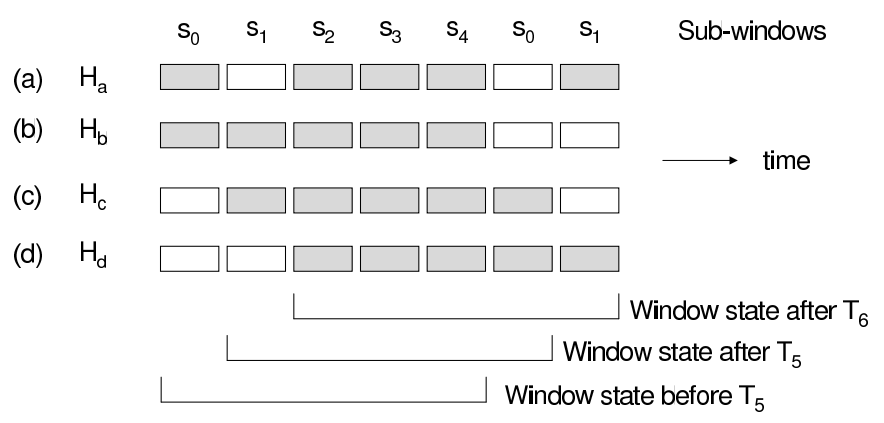

Figure 5: Differences in the results returned by $T_{Q 1}$ in $H_{a}, H_{b}, H_{c}$, and $H_{d}$.

reflects both $T_{5}$ and $T_{6}$ (recall Figure $5(\mathrm{~d})$ ). Again, this is because only $S G\left(H_{d}\right)$ serializes both window movements before $T_{Q k}$, meaning that $T_{Q k}$ sees both updates.

\subsection{Isolation Levels for Concurrent Execution of Queries and Window Move- ments}

Having shown that the serialization order affects the semantics of read-only transactions, we propose two stronger isolation levels that restrict the allowed serialization orders.

Definition 1. A serializable history $H$ is said to be window-serializable (WS) if all of its committed $T_{Q k}$ transactions read a true state of the sliding window as of some point in the past or present (i.e., a contiguous sequence of sub-windows is read, as in Figure 5 (b), (c), and (d)).

Definition 2. A window-serializable history $H$ is said to be latest-window-serializable (LWS) if all of its committed $T_{Q k}$ transactions read the state of the sliding window that reflects all the window update transactions that have committed before $T_{Q k}$ commits.

Note that $H_{a}$ is neither $W S$ nor $L W S, H_{b}$ and $H_{c}$ are $W S$ but not $L W S$, and $H_{d}$ is ( $W S$ and) $L W S$. Note that only $L W S$ guarantees that queries read the most up-to-date state of the window Motivated by Fig. 4, we prove the following equivalences between the above definitions and the resulting serialization graphs

Theorem 1. A history $H$ is window-serializable iff $S G(H)$ has the following property: for any $T_{Q k}$, if any $T_{i}$ is serialized before $T_{Q k}$, then for all $T_{j}$ serialized after $T_{Q k}, i<j$.

Proof. Suppose that $H$ is $W S$. If all transactions $T_{Q k}$ contained in $H$ incur at most one concurrent window movement, then clearly, $S G(H)$ satisfies the desired property. Otherwise, note that for $T_{Q k}$ to read a sliding window state from some point in the past or present, it must be the case that either $T_{Q k}$ is isolated from all the concurrent window updates, or it only reads the least recent update, or it only reads the two oldest updates, and so on. In all cases, $S G(H)$ contains less recent updates serialized before the query and more recent updates serialized after the query, as wanted. Now suppose that $S G(H)$ satisfies the property that all $T_{j}$ serialized after any $T_{Q k}$ have higher subscripts than those $T_{i}$ which are serialized before $T_{Q k}$. Let $m$ be the maximum subscript of any transaction $T_{i}$ serialized before $T_{Q k}$. It follows that $T_{Q k}$ reads a sliding window state that resulted from applying all the updates up to $T_{m}$ and therefore $H$ is WS.

Theorem 2. A history $H$ is latest-window-serializable iff $S G(H)$ has the following property: for any $T_{Q k}$, all concurrent $T_{i}$ transactions must be serialized before $T_{Q k}$. 
Proof. Suppose that $H$ is $L W S$ and let $T_{Q k}$ be any query that incurs at least one concurrent window movement. It follows that $T_{Q k}$ reads a state of the window that results from applying all the concurrent updates. Hence, concurrent window updates must be serialized before queries, as wanted. Now suppose that $S G(H)$ does not contain any links pointing from any $T_{Q k}$ to any $T_{i}$. This means that there are no queries that have been interrupted by window updates which the queries then did not see. Hence, $H$ is $L W S$.

\section{Transaction Scheduler Design}

\subsection{Producing $L W S$ Histories}

We now present the design of a DSMS transaction scheduler that produces $L W S$ histories. Recall from Section 2 that write-only transactions $T_{j}$ must be executed with highest priority (possibly by interrupting concurrently running read-only transactions) so that queries have access to an up-to-date version of the window. Given this assumption, our scheduler executes window movements optimistically and uses serialization graph testing (SGT) to abort any read-only transaction that causes a read-write conflict. In general, $S G T$ may suffer from high space usage and long running time if many conflicts among many transactions must be tracked over time [5]. Fortunately, in our context, the serialization graph is simple and can be pruned dynamically. In particular, for each currently running $T_{Q k}$, it suffices to monitor concurrent window movements $T_{j}$ and ensure that all interleaved $T_{j}$ are serialized before $T_{Q k}$ (recall Figure 4 ). Once $T_{Q k}$ commits, it is guaranteed not to cause $L W S$ violations at any point in the future, and therefore its node can be safely deleted from the serialization graph.

The scheduler is summarized as Algorithm 1. Lines 3 and 4 serially execute window movements immediately (technically, line 4 must wait for an acknowledgement that the write operation has been performed). Lines 8 through 11 initialize a bit array $B_{Q k}$ for each newly arrived $T_{Q k}$, where bit $i$ is set if $T_{Q k}$ has already read sub-window $i$. Lines 12 through 18 execute read-only transactions, one sub-window scan at a time, and set the corresponding bit in $B_{Q k}$ to true. Again, before committing $T_{Q l}$ in line 17, the algorithm must wait for an acknowledgement of performing the read operation from line 14. Note that Algorithm 1 allows multiple read-only transactions to be executed at the same time in any order (line 13) because they do not conflict with one another. Lines 5 through 7 resolve $L W S$ conflicts, as proven below.

Theorem 3. Algorithm 1 produces LWS histories.

Proof. As per Definition 2, we need to show that all committed read-only transactions $T_{Q k}$ have the property that any window movements $T_{j}$ that were executed at the same time as $T_{Q k}$ are serialized before $T_{Q k}$. First, note that the only time that a new $L W S$ violation may possibly appear is after a window update $T_{j}$ commits while one or more $T_{Q k}$ transactions are still running. Furthermore, a $L W S$ conflict appears only if any $T_{j}$ has updated a sub-window (an older copy of) which has already been read by any of the currently running $T_{Q k}$ transactions, in which case $T_{j}$ would be serialized before $T_{Q k}$. This occurs if $B_{Q k}[j \bmod n]$ is set for any currently running $T_{Q k}$. In this case, Algorithm 1 aborts $T_{Q k}$ (line 7 ), ensuring that all $T_{Q k}$ committed in line 17 satisfy Definition 2.

To see an example of aborting a transaction, recall history $H_{c}$ from the previous section and suppose that Algorithm 1 has processed the following prefix of it: 


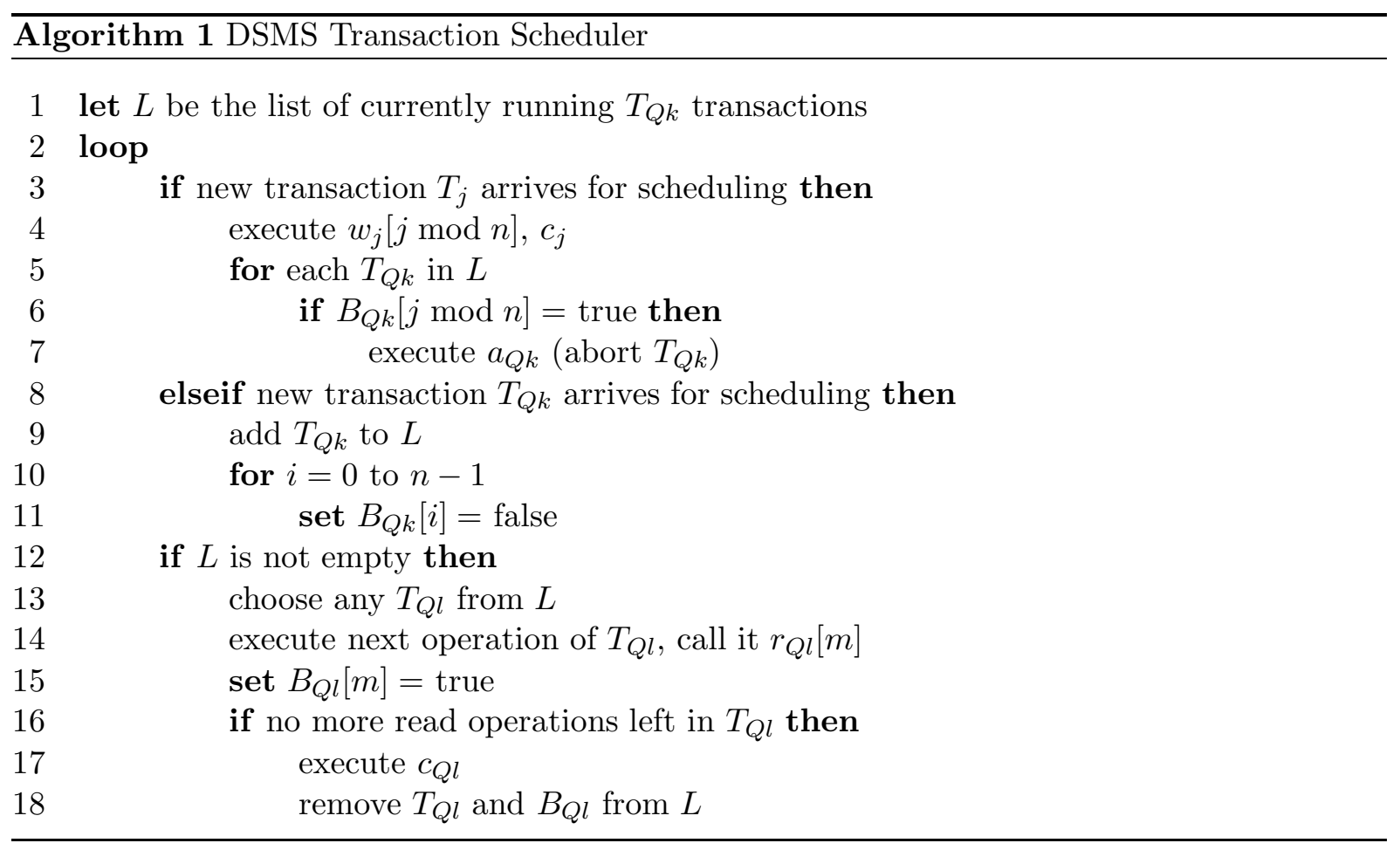

$H_{c}^{\prime}=r_{Q 1}[4] w_{5}[0] c_{5} r_{Q 1}[3] r_{Q 1}[2] r_{Q 1}[1] w_{6}[1] c_{6}$.

When $T_{6}$ commits, $B_{Q 1}[4], B_{Q 1}[3], B_{Q 1}[2]$, and $B_{Q 1}[1]$ are set because these sub-windows have already been read. Executing line 6 , we discover that $B_{Q 1}[6 \bmod 5]=B_{Q 1}[1]$ is true and therefore we abort $T_{Q 1}$ in line 7 . Similarly, in $H_{a}$ and $H_{b}, T_{Q 1}$ must be aborted immediately after $T_{5}$ commits.

Note that Algorithm 1 supports read-only transactions with different priorities, such as snapshot queries or "important" long-running queries (as in $Q_{1}$ from Figure 1 (d)). To do this, we assume that the query manager embeds a priority $p$ within each $T_{Q k}$ and we change line 13 in Algorithm 1 to read: "let $T_{Q l}$ be the transaction in $L$ with the highest value of $p$ ". Consequently, if a low-priority $T_{Q k}$ is currently being executed, then a higher-priority $T_{Q m}$ transaction has the effect of suspending $T_{Q k}$. This extension does not impact the correctness of Algorithm 1 as it does not introduce any new $L W S$ conflicts.

\subsection{Optimal Ordering of Read Operations}

Given that Algorithm 1 may abort read-only transactions in order to guarantee $L W S$, we want to minimize the required number of aborts. The idea is to shuffle the read operations within $T_{Q k}$ given the following insight. Since aborts occur when a sub-window is being updated but an older version of it has already been read by a concurrent $T_{Q k}$ transaction, we should execute $T_{Q k}$ by first reading the sub-window which is scheduled to be updated the farthest out into the 


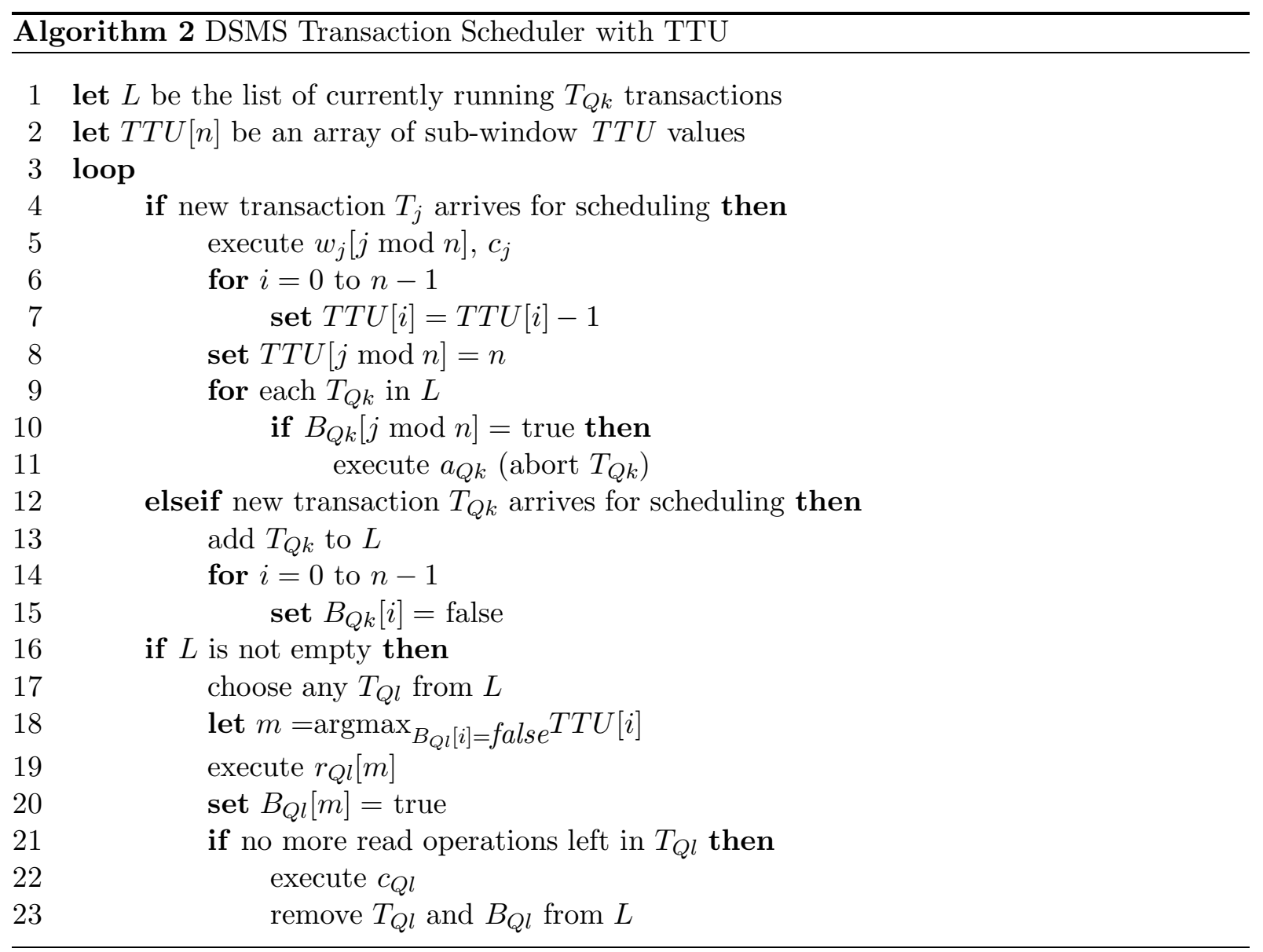

future. More precisely, we define the time-to-update (TTU) of a sub-window as the number of window-movement transactions $T_{j}$ that must be applied until this sub-window is updated. When the scheduler chooses a read-only transaction $T_{Q k}$ to process, it always executes the remaining read operation of $T_{Q k}$ whose sub-window has the highest $T T U$ value at the given time.

The revised scheduler is shown below as Algorithm 2 (again, adding support for multiple priority levels can be done by changing line 17 to process the highest-priority transaction). There are two main changes. First, lines 6 through 8 update the TTU values of each subwindow after every window movement. The newly updated sub-window receives a value of $n$ (it will take $n$ write-only transaction until this sub-window is updated again), whereas the TTU values of the remaining sub-windows are decremented. Furthermore, line 18 selects $m$ to be the index of the sub-window which has the highest $T T U$ value and has not been read by $T_{Q l}$.

The idea in Algorithm 2 is similar to the Longest Forward Distance (LFD) cache replacement algorithm [4], which always evicts the page whose next access is latest. LFD is optimal in the off-line case in terms of the number of page faults, given that the system knows the entire page request sequence and that all page faults have the same cost.

Theorem 4. Algorithm 2 is optimal for ensuring $L W S$ in the sense that it performs the fewest possible aborts for any history $H$. 
Proof. Let $A$ be the transaction scheduler in Algorithm 2 and let $S$ be any other transaction scheduler that serializes transactions in exactly the same way as $A$, but only differs in the ordering of read operations inside one or more read-only transactions. That is, $S$ corresponds to Algorithm 1 with some arbitrary implementation of the meaning of "next operation" in line 14. We need to prove that $S$ performs no fewer aborts than $A$ for any history $H$. Let $H_{i}$ be the prefix of $H$ containing the first $i$ read operations (interleaved with zero or more write operations, and zero or more commit or abort operations). The proof proceeds by inductively transforming the sequence of read operations produced by $S$ into that produced by $A$, one read operation at a time. To accomplish this, we let $S_{0}=S$ and define a transaction scheduler $S_{i+1}$ that, given $S_{i}$, has the following two properties.

1. Both $S_{i}$ and $S_{i+1}$ order all the read operations in $H_{i}$ in the same way as $A$.

2. $S_{i+1}$ orders all the read operations in $H_{i+1}$ in the same way as $A$ and performs no more aborts than $S_{i}$ in $H_{i+1}$.

Let $r_{k}[y]$ be the $(i+1)$ st read operation executed by $S_{i}$ and $r_{k}[z]$ be the $(i+1)$ st read operation executed by $S_{i+1}$. Due to our assumption that $A$ and $S$ only differ in the ordering of read operations inside read-only transactions, the $(i+1)$ st read operations done by $S_{i}$ and $S_{i+1}$ both belong to the same transaction, call it $T_{Q k}$. Thus, sub-window $z(\bmod n)$ has the highest TTU value at this time. Now, if $z=y$ then $S_{i+1}=S_{i}$ and we are done (property 2 holds). Otherwise, $S_{i+1}$ and $S_{i}$ differ in the $(i+1)$ st read operation. First, suppose that $T_{Q k}$ is not interrupted by any write-only transactions before the next read operation. Then, $T_{Q k}$ is not aborted by $S_{i}$ or by $S_{i+1}$ in $H_{i+1}$ and we are done (property 2 holds). Next, suppose that $T_{Q k}$ is interrupted by at least one write-only transaction before the next read operation. The remainder of the proof is broken into the following three cases, which collectively prove property 2 .

In the first case, suppose that the set of interrupting transactions contains $T_{y}$, but not $T_{z}$. Given that sub-window $z(\bmod n)$ has the highest TTU value at this time, and that write-only transactions are generated and serially executed in increasing order of their subscripts, the most recent write-only transaction can have a subscript no higher than $z-1$. Then, $S_{i}$ aborts $T_{Q k}$ in $H_{i+1}$. This is because $T_{Q k}$ has already read an old version of sub-window $y(\bmod n)$ and therefore $T_{y}$ would have been serialized after $T_{Q k}$. However, $S_{i+1}$ does not abort $T_{Q k}$ in $H_{i+1}$. To see this, observe that $T_{Q k}$ could not have possibly read any of the sub-windows that have just been updated. This is due to the fact that those sub-windows must have lower TTU values than sub-window $z(\bmod n)$ and must necessarily be scheduled after sub-window $z(\bmod n)$ by $S_{i+1}$.

In the second case, suppose that the set of interrupting transactions does not contain $T_{y}$ or $T_{z}$. By the same reasoning as above, the most recent write-only transaction can have a subscript no higher than $y-1$. Again, $S_{i+1}$ does not abort $T_{Q k}$ in $H_{i+1}$ because $T_{Q k}$ could not have possibly read any of the sub-windows updated by or before $T_{y-1}$ (they all have lower $T T U$ values than sub-window $z(\bmod n)$. In terms of satisfying property 2 , it does not matter what $S_{i}$ does in this case.

Finally, in the third case, suppose that the set of interrupting transactions contains both $T_{y}$ and $T_{z}$. Then, both $S_{i}$ and $S_{i+1}$ abort $T_{Q k}$ in $H_{i+1}$ because both schedulers have allowed $T_{Q k}$ 
to read a sub-window that has now been updated.

Algorithm 2 takes advantage of the semantics of DSMS transactions in order to anticipate possible $L W S$ violations and prevent them to the greatest possible extent. As an example, we trace the scheduling of the read operations of $T_{Q 1}$ in history $H_{d}$ defined in Section 3. At the beginning, sub-window zero is the oldest and has a TTU of one (it will be updated after the next write transaction, namely $T_{5}$ ). Sub-window one is next with a TTU of two, and so on until sub-window four, which has a TTU of five. Thus, the first read operation to be scheduled reads the sub-window with the highest $T T U$ value, namely sub-window four. Next, sub-window zero is updated and therefore its TTU changes to five; the TTU values of all the other sub-windows are decremented. Thus, sub-window zero is the next one to be read by $T_{Q 1}$ because its $T T U$ value of five is the highest. Sub-windows three and two are next (with TTU values of three and two, respectively). At this point, only sub-window one remains to be read, which is done after it is updated by $T_{6}$.

Thus far, we implicitly assumed that newly arrived data overwrite the oldest sub-window, meaning that a query which has read an old copy of a freshly updated sub-window must be aborted to guarantee $L W S$. Recall our discussion in Sect 2.2, mentioning the possibility of temporarily keeping expired sub-windows and allowing queries to re-read them in order to "undo" the contribution of expired tuples from the query state. Algorithm 2 still applies in this case. The difference is that when a $L W S$ conflict is discovered, the transaction re-scans the old copy of the newly updated sub-window, followed by reading the new copy. Therefore, no aborts are necessary. In this context, Algorithm 2 is optimal in the sense of minimizing the number of required re-scans.

\subsection{Note on Queries Accessing a Materialized Sub-Result}

Up to now, we assumed that queries access individual windows or their summaries. However, as discussed in [16], all but the simplest sliding window queries may re-order tuples during processing, meaning that the order in which tuples are inserted into a materialized sub-result may be different than the expiration order. As a consequence, it is no longer the case that the oldest sub-window may be dropped and the newest sub-window inserted in its place. Consider a join of two windows, each having five sub-windows. The result of the join may also be maintained using five sub-windows partitioned on expiration time. As illustrated in Figure 6, when the underlying windows slide, the oldest sub-window expires, as before. However, each sub-window may incur insertions because the new tuples may have various expiration times. To deal with this issue, we make a small modification to our scheduling algorithms: when a materialized sub-result is updated while a query is scanning it (or a summary over it), the tuples inserted into sub-windows which have already been read are copied and passed to the query prior to being inserted into their sub-windows (and summaries). This way, the query is guaranteed to see all the updates and $L W S$ is preserved. Note that the query need not read new tuples that were inserted into sub-windows which it has not read yet (they will be read when the query finally scans these sub-windows). 


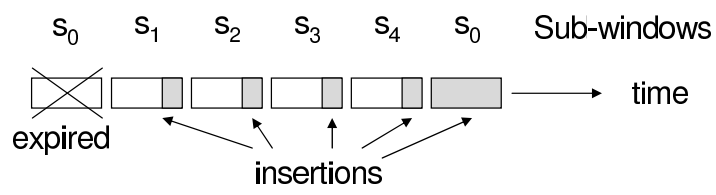

Figure 6: Update of a materialized sub-result.

\section{Experiments}

\subsection{Implementation Details and Experimental Procedure}

We implemented a simple DSMS query manager and the following four transaction schedulers: Algorithm 2 (abbreviated TTU), Algorithm 1 (which does not re-order the read operations within transactions, abbreviated $L W S$ ), a scheduler similar to Algorithm 2 that only enforces window-serializability (abbreviated $W S$ ), and a scheduler that executes transactions serially (as in current DSMSs, abbreviated Serial). The implementation was done in Java (Blackdown $J a v a^{T M}$, J2SE 1.4.2), while the experiments were performed on a Pentium-IV PC with a 3 $\mathrm{GHz} \mathrm{CPU}$ and $1 \mathrm{~Gb}$ of RAM, running Linux. The input stream is a sequence of simulated IP header packets, with the expected fields such as timestamp, protocol, source and destination IP addresses, header size, data size, TCP flags, time-to-live, and checksum. For simplicity, the values of all fields are generated randomly. For example, the source and destination IP addresses have one of one thousand random values, whereas the data size is a random integer between one and 100. The steady-state stream arrival rate is one packet per millisecond, but the specific arrival rate over a particular sub-window is allowed to deviate from the steady-state rate by a factor of up to ten.

We use a long-running query workload representative of an on-line network traffic analysis application (see, e.g., [9, 11]). There are two levels of transaction priorities: those corresponding to re-executions of long-running queries with low-priority, and those corresponding to snapshot queries with high-priority. We execute a total of between 40 and 100 long-running queries, arranged into groups of five for shared processing. Long-running queries are chosen randomly from the following set: top- $k$ queries over the source or destination IP addresses, and percentile queries (25th, 50th, 75th, 90th, 95th, and 99th percentiles) over the total bandwidth consumed by (or directed to) distinct IP addresses. The window sizes referenced by queries are generated randomly between one and $n$, where $n$ is the total number of sub-windows. Queries computing the same aggregate over different window sizes are evaluated together. For simplicity of implementation, long-running queries are executed by scanning the window and building a hash table on the required attribute. Snapshot queries are chosen from a set of simple aggregates over a random subset of the source and destination IP addresses. Each query references the same time-based window, which is stored in main memory.

The experimental procedure is as follows. After initializing the sliding window using a randomly generated input stream, we test each of the four transaction schedulers over an identical query workload. The tests proceed for a time equal to the window length. We then repeat each test five times using different input streams and calculate the average of each measurement being reported. The parameters being varied in (and across) the experiments are the query workload, 


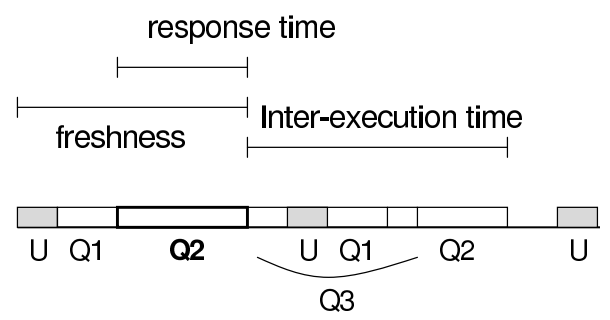

Figure 7: Freshness, response time, and inter-execution time of $Q_{2}$.

the window size (controlled via the number of sub-windows), and the length of each sub-window (which controls the frequency of window movements). The following performance metrics are used to evaluate the four transaction schedulers, as illustrated in Figure 7 corresponding to the execution time line from Figure 1 (d).

- Query freshness is the difference between the time that a query reports an answer and the time of the last window update reflected in the answer.

- Response time is the difference between the query execution start time and end time. This metric is particularly important for snapshot queries, which are usually time-sensitive.

- Inter-execution time of a long-running query is the length of the interval between its reexecutions. A DSMS is expected to tolerate slightly longer inter-execution times if the returned answers are more up-to-date. The motivation for this is that even if we return an older answer earlier, we would have to re-execute the query soon in order to produce an answer that reflects the new state of the window.

\subsection{Percentage of Aborted Transactions}

Our first experiment illustrates Theorem 4 by comparing the percentage of aborted read-only transactions using Algorithms 1 and 2. We test two sub-window sizes: $t=1 \mathrm{sec}$. and $t=5 \mathrm{sec}$, with the number of sub-windows varied from ten to 100. The number of long-running queries is set to 40 for $t=1 \mathrm{sec}$. and 100 for $t=5 \mathrm{sec}$. The percentage of transactions aborted by Algorithm 1 is shown in Figure 8. In comparison, Algorithm 2 did not abort any transactions in any of the experiments. This is because during normal execution, a long-running query does not incur more than one concurrent window update, unless suspended for a long time in order to run a heavy workload of snapshot queries. Since Algorithm 2 ensures that read-only transactions postpone reading the sub-window that is about to be updated until the end, aborts can be easily avoided if the number of concurrent window updates is small.

Note that the proportion of read-only transactions aborted by Algorithm 1 is higher for $t=1$ sec. because a smaller sub-window size implies that window movements are executed more often, thereby increasing the chances of read-write conflicts. Aborts are also more frequent as the number of sub-windows increases (i.e., as the length of the sliding window grows) because this causes longer query evaluation times, therefore the number of read-only transactions between window updates decreases. In turn, this increases the proportion of read-only transactions that 


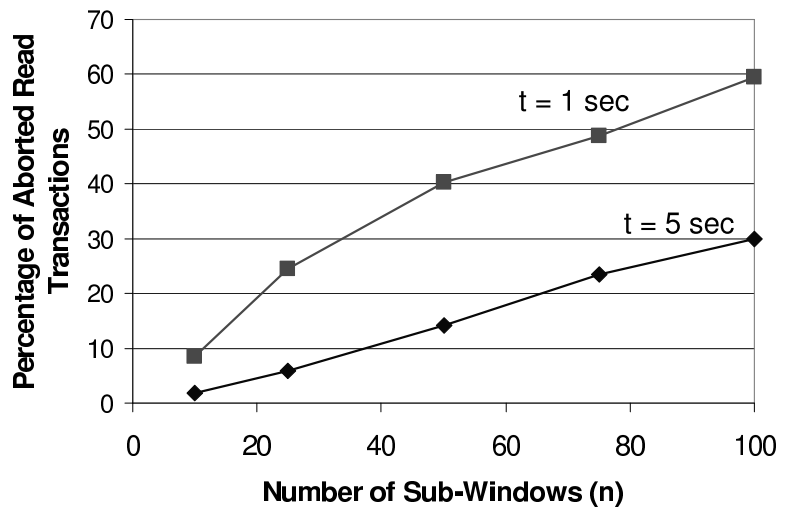

Figure 8: Percentage of read-only transactions aborted by Algorithm 1.

execute at the same time as window movements and raises the chances of read-write conflicts. In the worst case of frequent window movement and long window size $(t=1 \mathrm{sec}$. and 100 sub-windows), Algorithm 1 aborts over half of the read-only transactions because nearly every transaction incurs a concurrent window movement, which often ends up causing a $L W S$ conflict. Similar results were obtained when snapshot queries were posed at random times. In particular, Algorithm 2 still did not abort any transactions.

\subsection{Experiments with a Workload of Long-Running Queries}

Next, we present results of executing Serial, WS, LWS, and TTU on a workload consisting of long-running queries and interleaved window movements. The sub-window sizes, number of sub-windows, and number of long-running queries are the same as in the previous experiment; for now, we assume that no snapshot queries are posed. We measure the average freshness, inter-execution time, and throughput.

The average query freshness, in units of seconds, is shown in Figure 9 (the lower the value, the better). TTU and $L W S$ clearly outperform $W S$ and Serial because the first two guarantee latest-window-serializable schedules, where queries have access to an up-to-date state of the window. As expected, freshness deteriorates for all four schedulers as the sub-window size grows to $t=5 \mathrm{sec}$. and window movements become less frequent. Moreover, increasing the number of sub-windows (or equivalently, increasing the window length) generally has an adverse effect on freshness because the query execution times increase. Note that Serial performs slightly better than $W S$ because $W S$ adds to the query execution time by performing concurrent window movements, yet the answer does not reflect any of the updates. Overall, TTU provides the best query freshness in all tested scenarios.

The average query inter-execution times, in units of seconds, are graphed in Figure 10. Each cluster of eight bars corresponds, in order, to Serial, WS, LWS, and TTU for $t=1$ sec., followed by Serial, WS, $L W S$, and TTU for $t=5 \mathrm{sec}$. Serial has the best (lowest) inter-execution times because it does not incur the overhead of serialization graph testing, therefore its total query execution time is slightly lower. Notably, LWS (corresponding to the third and seventh bars in each cluster) performs the worst. For instance, aborting every second re-execution of 


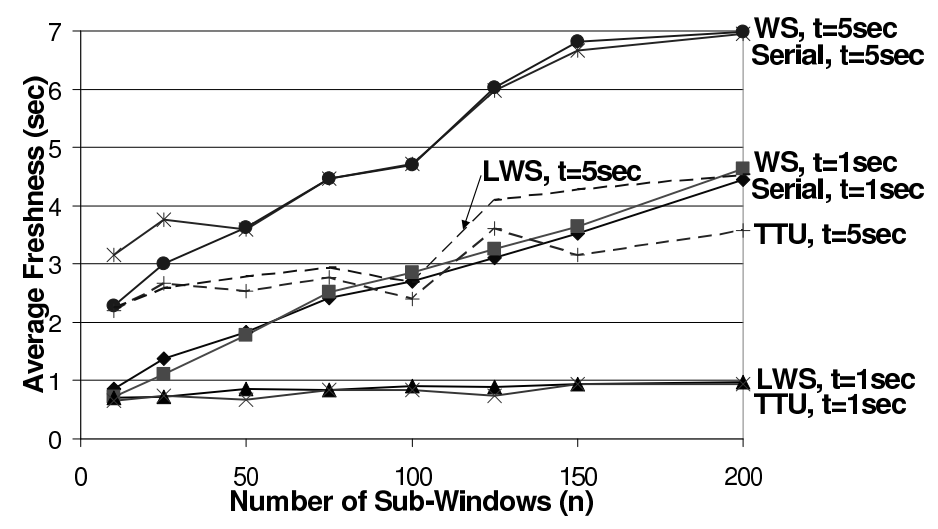

Figure 9: Comparison of query freshness for Serial, WS, LWS, and TTU.

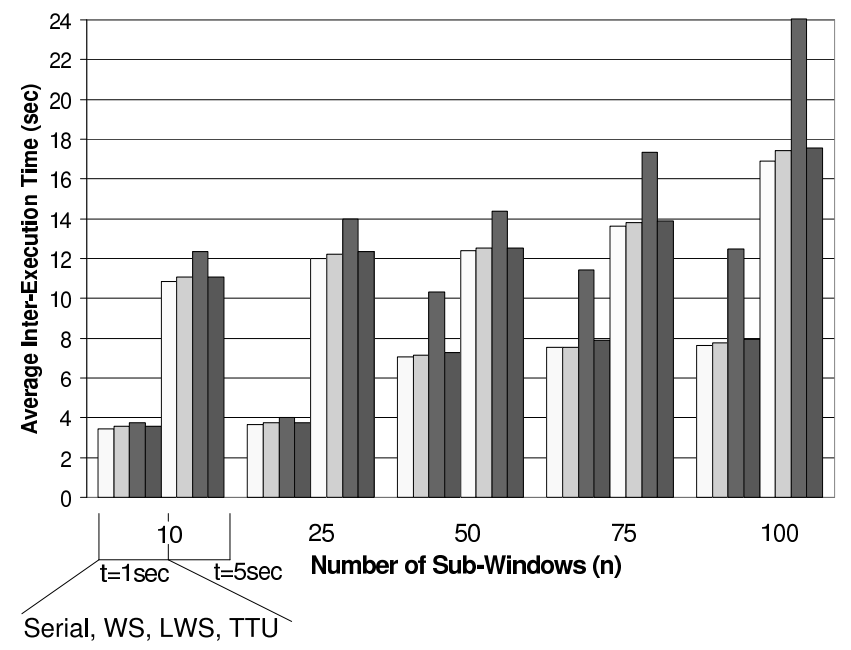

Figure 10: Comparison of query inter-execution times for Serial, WS, LWS, and TTU.

a long-running query means that its inter-execution time doubles. In general, increasing the sub-window size to $t=5 \mathrm{sec}$. (and hence, increasing the total window size) leads to longer interexecution times for all four schedulers as queries take longer to process. Similarly, increasing the number of sub-windows increases the query evaluation times and therefore negatively affects the inter-execution times. Overall, Serial yields the best query inter-execution times, with $W S$ and $T T U$ following very closely behind, whereas $L W S$ performs badly due to aborted transactions.

Figure 11 illustrates the throughput (in units of the number of read-only transactions per second) of $L W S$ versus the other three schedulers (namely Serial, WS, and TTU, labeled Others), which all yield very similar results. In particular, the throughput penalty of TTU versus Serial is very small - typically below two percent and at most four percent. This is because the serialization graph testing done by $T T U$ consists of simple bit operations after each window movement and causes negligible overhead. Note the poor performance of $L W S$; its throughput is lower in all cases due to aborted transactions. As expected, the throughput of all schedulers 


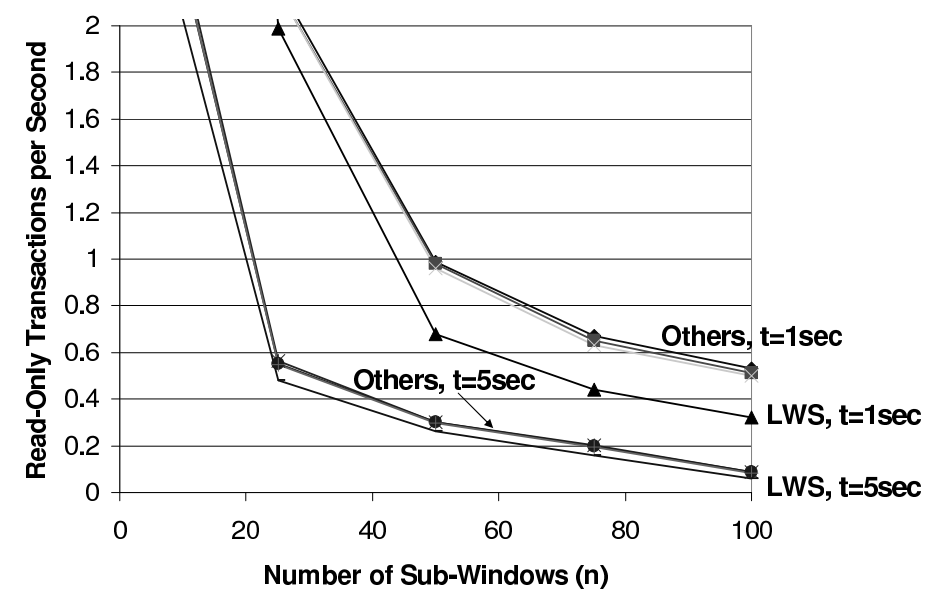

Figure 11: Comparison of throughput of read-only transactions for LWS and "Others".

decreases when transactions take longer to execute, which occurs when the sliding window is large (i.e., the number of sub-windows increases or the sub-window size increases).

\subsection{Experiments with a Workload of Long-Running and Snapshot Queries}

Last, we report the results of experiments with a mixed workload of long-running and snapshot queries (and concurrent window movements). We fix the sub-window size at five seconds, the number of long-running queries at 100 , and the number of snapshot queries per sub-window length at five. Snapshot queries are scheduled at random times with an average time between requests set to one second. We report the average snapshot query response time, and we separately measure the average freshness of snapshot and long-running queries.

We begin by analyzing the average snapshot query response times, in units of seconds, shown in Figure 12. TTU and $W S$ perform best and yield nearly identical response times. The response times of $L W S$ are noticeably longer because it is forced to abort and restart some snapshot queries. Thus, LWS may not be suitable for a DSMS that executes time-sensitive queries. Serial exhibits the worst results in this experiment because it is unable to suspend a longrunning query and execute an snapshot query immediately; in general, Serial is inappropriate for any situation involving prioritized scheduling. As the number of sub-windows increases, the response time achieved by each of the four schedulers worsens because it is now more costly to execute each query.

Figure 13 plots the average snapshot query freshness, in units of seconds. As expected, $T T U$ outperforms the other schedulers because it guarantees latest-window-serializability and does not abort any transactions. The performance of $L W S$ is somewhat worse because some of the transactions corresponding to snapshot queries are aborted and restarted at a later time. $W S$ and Serial do not guarantee latest-window serializability and therefore exhibit the worst performance. Overall, TTU yields the best results in terms of snapshot query freshness and is tied for best in terms of the response time.

Finally, we separately examine the average freshness of long-running queries in order to verify that the performance edge of $T T U$ in the context of snapshot query freshness does not come at 


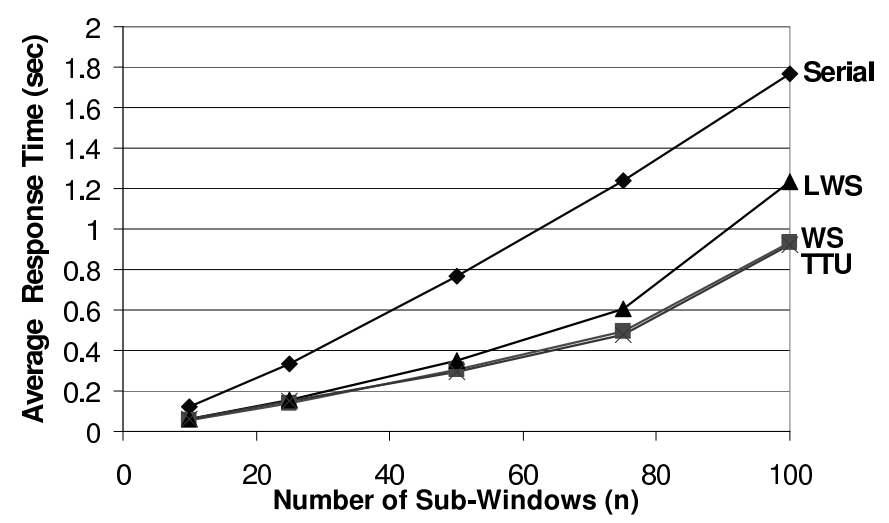

Figure 12: Comparison of snapshot query response times for Serial, WS, LWS, and TTU.

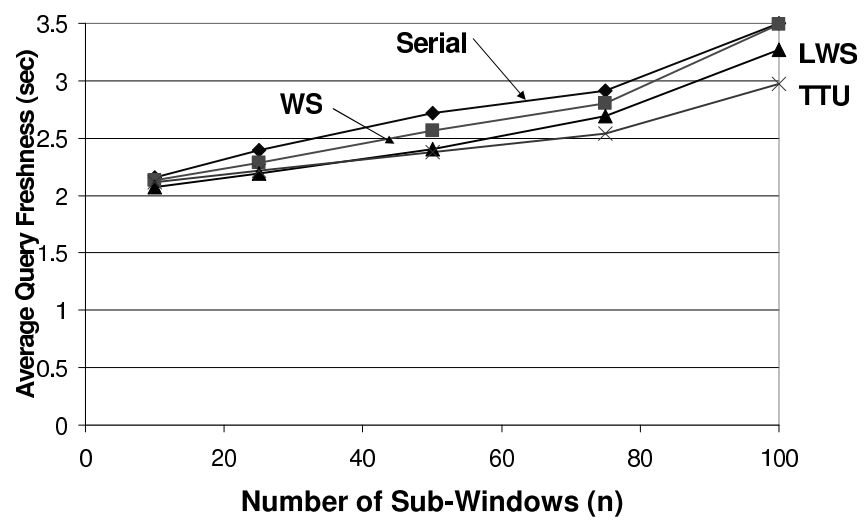

Figure 13: Comparison of snapshot query freshness for Serial, WS, LWS, and TTU.

a cost of poor long-running query freshness. Results are shown in Figure 14. It can be seen that $T T U$ maintains its superiority in producing the most up-to-date results of long-running queries.

\section{Comparison with Related Work}

The concurrency control mechanisms presented in this paper are compatible with any DSMS that employs periodic updates of sliding windows and query results, e.g., [1, 2, 6, 8, 14, 17, 19]. Our techniques are also applicable to a system such as PSoup [7], where mobile users connect to a DSMS intermittently and retrieve the latest results of sliding window queries. In our context, these asynchronous requests may be modeled as snapshot queries posed at various times. Given that mobile users may have low connectivity with the system (e.g., via a wireless channel), it is particularly important to guarantee low response times and up-to-date query answers. Our transaction scheduler fulfills both of these requirements.

As discussed in Section 2, we assumed an evaluation model in which queries are re-executed by scanning one or more windows or summaries, or a materialized sub-result. Similar techniques were used in $[3,14,19]$. Our procedure for incremental maintenance of materialized join results- 


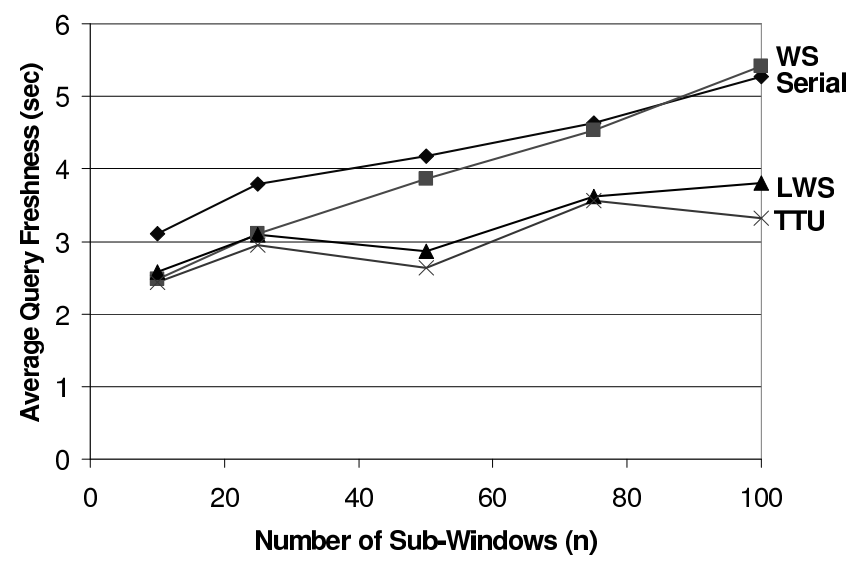

Figure 14: Comparison of long-running query freshness for Serial, WS, LWS, and TTU.

using a batch of newly arrived tuples from one window to probe the other window and generate new results - is similar to the lazy multi-way join from [15]. In general, our query model of a final aggregation function applied on a window or materialized sub-result is similar to NiagaraCQ [8], but less expressive than, e.g., Aurora [1] and STREAM [2]. However, we believe that our model is sufficiently expressive for many applications that require long-running queries for monitoring purposes, while at the same time being simple enough to allow straightforward solutions of concurrency control issues.

Our transaction model resembles multi-level concurrency control and multi-granularity locking as it considers a sub-window, rather than an entire window, to be an atomic data object. The novelty of our solution is that the order in which read operations are performed is chosen in such a way as to minimize the number of aborted transactions.

Our transaction scheduler employed serialization graph testing. Other scheduling techniques include two-phase locking and timestamping [5]. However, two-phase locking may not be appropriate in our context because it is not clear how to force a particular serialization order using locks. Moreover, the possible problem with using timestamping for DSMS concurrency control is the difficulty of ensuring latest-window serializability. Suppose that each transaction receives a timestamp when it is passed to the transaction scheduler and that serialization order is determined by timestamps. In this case, any concurrent window update transaction is assigned a higher timestamp than a read-only transaction and is therefore serialized before the read-only transaction. Hence, Algorithm 2 would be forced to abort every read-only transaction that is interrupted by a window movement. A similar issue appears if we want to adapt multi-versioning concurrency control techniques to enforce latest-window serializability, among them snapshot isolation and commit-order preserving serializability [18].

\section{Conclusions and Future Work}

This paper presented DSMS concurrency control mechanisms that allow a window to slide forward while it, or an associated summary structure, is being scanned by a query. Our solution is 
based upon a model that views DSMS data access as a mix of concurrent read-only and write-only transactions. We proved that conflict serializability is insufficiently strong to guarantee correct and up-to-date query results, and defined more appropriate isolation levels. We also implemented a transaction scheduler for enforcing the new isolation levels that is provably optimal in reducing the number of aborted transactions. Our scheduler was experimentally shown to improve query freshness and response times while maintaining high transaction throughput.

We are interested in the following two directions for future work. First, we want to extend our query execution model and investigate concurrency control issues in query plans containing an arbitrary number of pipelined window operators. One issue in this context is synchronization among the levels in the pipeline, e.g., updates to the individual windows may take some time as they are propagated up the pipeline to the final query operator. Another problem appears when the same sub-query occurs more than once within a query, in which case our current assumption of queries scanning each window once may not hold (unless the sub-query can be flattened). Second, we want to extend our treatment of DSMS concurrency control to include the semantics of data loss and crash recovery, e.g., loss of data for a particular time interval, which might make it impossible for queries to read a full window.

\section{References}

[1] D. Abadi, D. Carney, U. Cetintemel, M. Cherniack, C. Convey, S. Lee, M. Stonebraker, N. Tatbul, and S. Zdonik. Aurora: A new model and architecture for data stream management. The VLDB Journal, 12(2):120-139, Aug 2003.

[2] A. Arasu, S. Babu, and J. Widom. The CQL continuous query language: Semantic foundations and query execution. The VLDB Journal, 14(1), 2005, to appear.

[3] A. Arasu and J. Widom. Resource sharing in continuous sliding-window aggregates. In Proc. 30th Int. Conf. on Very Large Data Bases, pages 336-347, 2004.

[4] L. A. Belady. A study of replacement algorithms for virtual storage computers. IBM Syst. J., 5:78-101, 1966.

[5] P. Bernstein, V. Hadzilacos, and N. Goodman. Concurrency Control and Recovery in Database Systems. Addison-Wesley, 1987.

[6] S. Chandrasekaran, O. Cooper, A. Deshpande, M. J. Franklin, J. M. Hellerstein, W. Hong, S. Krishnamurthy, S. Madden, V. Raman, F. Reiss, and M. Shah. TelegraphCQ: Continuous dataflow processing for an uncertain world. In Proc. 1st Biennial Conf. on Innovative Data Syst. Res., pages 269-280, 2003.

[7] S. Chandrasekaran and M. J. Franklin. PSoup: a system for streaming queries over streaming data. The VLDB Journal, 12(2):140-156, Aug 2003.

[8] J. Chen, D. DeWitt, F. Tian, and Y. Wang. NiagaraCQ: A scalable continuous query system for internet databases. In Proc. ACM SIGMOD Int. Conf. on Management of Data, pages 379-390, 2000. 
[9] G. Cormode, T. Johnson, F. Korn, S. Muthukrishnan, I. Spatscheck, and D. Srivastava. Holistic UDAFs at streaming speeds. In Proc. ACM SIGMOD Int. Conf. on Management of Data, pages 35-46, 2004.

[10] G. Cormode and S. Muthukrishnan. An improved data stream summary: The count-min sketch and its applications. In Proc. Latin American Theoretical Informatics (LATIN), pages 29-38, 2004.

[11] C. Cranor, T. Johnson, O. Spatscheck, and V. Shkapenyuk. Gigascope: High performance network monitoring with an SQL interface. In Proc. ACM SIGMOD Int. Conf. on Management of Data, pages 647-651, 2003.

[12] P. Flajolet and G. N. Martin. Probabilistic counting. In Proc. IEEE Conf. on Foundations of Computer Science, pages 76-82, 1983.

[13] L. Golab, D. DeHaan, E. Demaine, A. Lopez-Ortiz, and J. I. Munro. Identifying frequent items in sliding windows over on-line packet streams. In Proc. ACM SIGCOMM Internet Measurement Workshop, pages 173-178, 2003.

[14] L. Golab, S. Garg, and M. T. Özsu. On indexing sliding windows over on-line data streams. In Advances in Database Technology - EDBT'04, pages 712-729, 2004.

[15] L. Golab and M. T. Özsu. Processing sliding window multi-joins in continuous queries over data streams. In Proc. 29th Int. Conf. on Very Large Data Bases, pages 500-511, 2003.

[16] L. Golab and M. T. Özsu. Update-pattern aware modeling and processing of continuous queries. In Proc. ACM SIGMOD Int. Conf. on Management of Data, pages 658-669, 2005.

[17] N. Shivakumar and H. García-Molina. Wave-indices: indexing evolving databases. In Proc. ACM SIGMOD Int. Conf. on Management of Data, pages 381-392, 1997.

[18] G. Weikum and G. Vossen. Transactional Information Systems. Theory, Algorithms, and the Practice of Concurrency Control and Recovery. Morgan Kauffman, 2002.

[19] Y. Zhu and D. Shasha. StatStream: Statistical monitoring of thousands of data streams in real time. In Proc. 28th Int. Conf. on Very Large Data Bases, pages 358-369, 2002. 